\title{
Open Circuit Voltage of Lithium-ion Batteries for Energy Storage in DC Microgrids
}

\author{
Federico Baronti*, Roberto Saletti*, Walter Zamboni ${ }^{\S}$ \\ *Dip. di Ingegneria dell'Informazione - Università di Pisa, Italy \\ ${ }^{\S}$ Dip. di Ingegneria dell'Informazione, Ingegneria Elettrica e Matematica Applicata - Università degli Studi di Salerno, Italy \\ E-mail: roberto.saletti@unipi.it
}

\begin{abstract}
Rechargeable batteries, particularly Lithium-ion ones, are emerging as a solution for energy storage in DC microgrids. This paper reviews the issues faced in the characterization of the Open Circuit Voltage (OCV) of a Lithium-ion battery, starting from the problem of $\mathrm{OCV}$ measurement and ending with the modeling of OCV hysteresis. An accurate OCV modeling is necessary for a reliable estimation of the internal battery states, such as State-of-Charge and State-of-Health. These state variables are useful for a better control and a more efficient utilization of the energy storage system in the microgrid. We also compare with experiments two models that account for the hysteresis in Lithium-Iron-Phosphate batteries.
\end{abstract}

\section{INTRODUCTION}

DC microgrids are emerging as a possible successful scenario in the energy field, in which the balance between the generation of energy via renewable sources and the energy consumption is efficiently achieved. Often, energy is produced and consumed in DC electrical form. Thus, it makes sense to think of a network of generation/distribution/utilization where energy never leaves its DC electrical form, avoiding the necessity of back and forth conversions to the AC electrical form and the related power losses. On the one side, generation of energy occurs in many cases via renewable sources, such as photovoltaic panels, which produce DC electrical energy. This energy is presently converted to the $\mathrm{AC}$ form to be inserted in the distribution grid. On the other hand, many loads that use energy are DC loads that are supplied by rectifying and converting to the appropriate level the $\mathrm{AC}$ voltage of the distribution grid. This is particularly true for office and home appliances (e.g. computers, laptops, tablets, phones, printers, TVs, microwave ovens and lighting) that consume electricity in DC form [1].

DC microgrids are thus assuming more importance in local scenarios, in "islands" in which local generation and consumption of energy occurs, even when the island is disconnected from the general distribution grid, making the island autonomous, at least in principle. In this cases, always leaving the energy in its DC electrical form leads to higher efficiency. Unfortunately, the rate of energy generation from, say, photovoltaic panels is strongly dependent on the weather and illumination conditions, so that the energy generation rate is unreliable in time and intensity, as it also happens for the loads that follow an average daily general behavior. These considerations weaken the microgrid concept described above and the possible advantages that can be achieved. The solution that matches the unreliable and mostly unpredictable energy generation and consumption rates is the availability of Energy Storage Systems (ESSs) [2] inside the "island" that accumulate the excess energy in some time intervals and give it back to the loads when the energy production is insufficient. An efficient energy storage system is thus the key to exploit the DC microgrid potential.

If a DC microgrid is thought as a local entity that encompasses a very limited geographical area (i.e. even one or a few buildings), the most appropriate energy storage system technology is the electrochemical storage, realized by rechargeable batteries, particularly those based on the lithium-ion chemistry. These batteries are now leaving the low-power applications (mainly portable electronics), in which they firstly have been introduced, and penetrating the market of middle-power applications, such as electrical vehicles and even stationary energy storage systems. The enormous benefit of lithium-ion batteries with respect to the old lead-acid ones is the better energy and power densities in both volume and weight and the larger number of charge/discharge cycles sustainable. Lithium-ion energy storage systems for residential applications are thus foreseen to be feasible and affordable in the very near future [3]-[5].

\section{LITHIUM-ION BATTERY ENERGY STORAGE SYSTEM}

An energy storage system based on lithium-ion batteries may be much more efficient than its lead-acid counterpart, as the lithium-ion battery cell is more performing. Besides the already mentioned features (specific and volumetric density, useful cycles) lithium-ion cells also stand for the high charge and discharge current rates they can sustain. In many cases we find cells capable of being discharged at a current value of 1-10 C, being $\mathrm{C}$ the nominal cell charge capacity expressed in amperehour [6].

Unfortunately, lithium-ion cells also suffer from some issues that have to be solved before their successful utilization. First of all, safety is a fundamental issue, as overvoltage, undervoltage or overtemperature may cause damages to the cells and even fires. Therefore, lithium-ion batteries must be accurately monitored and controlled to avoid their use outside the proper operating range. A lithium-ion battery is always accompanied by an electronic system called Battery management System (BMS) which is in charge of monitoring and safely managing the battery [7]. Second, an accurate 


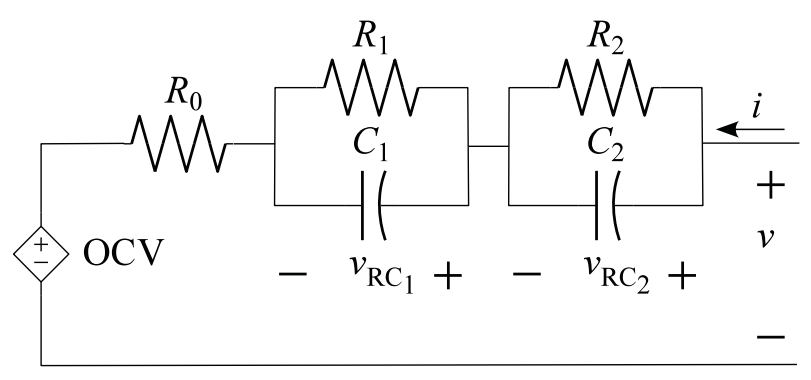

Figure 1. Electrical equivalent model of a lithium-ion cell, based on the Randle's model for the interfacial electrochemical reactions. OCV accounts for the cell's electromotive force.

model of the physical behavior of the lithium-ion cell may be fundamental for a better exploitation of its performance. Accurately knowing the inner status of a cell allows the knowledge of many parameters useful for the application: as an example the knowledge of the State-of-Charge (SoC) allows one to know the cell's residual charge and thus the expected operating time of the application. Knowing the State-of-Health $(\mathrm{SoH})$ allows one to have reasonable estimation of the ageing of the battery, of its loss of performance and an expectation of its residual useful life. Therefore, an accurate model of the battery is mandatory for controlling and exploiting ESSs at best [8]-[10].

\section{LITHIUM-ION CELL MODELS}

The most popular models of a lithium-ion cell are circuit models in which an electrical equivalent circuit tries to emulate the real voltage and current behavior of a battery cell. These models are simpler than electrochemical models and are thus rather easily implementable on the hardware platforms that control the ESS. One of the most popular model is based on the Randle's model for the interfacial electrochemical reactions and consists of a voltage power source that accounts for the Open-Circuit-Voltage (OCV) of the cell, followed by a series resistance and a series of one or more parallel resistancecapacitance groups that accounts for the dynamic effects that show up in the battery voltage behavior [11], [12], as its shown in Fig. 1. The OCV power source provides the cell's electromotive force that varies with the SoC according to a law specific to each particular variant of the lithium-ion chemistry. The OCV-SoC relationship is often considered invariant if normalized to the actual battery capacity [13]. The other model parameters can be extracted by experimental tests (e.g. Pulsed Current Tests, [14]), with which each circuit element value is calculated by fitting the measured voltage response in time with combinations of exponential terms.

Another method to derive the parameters of the cell model is the Electrochemical Impedance Spectroscopy (EIS), an experimental procedure with which the inner impedance of the cell is measured as a function of the frequency. Analysis of the EIS data leads to the circuit parameter values [15]. In any case, the equivalent circuit component parameters are found to be dependent on the battery operating conditions, such as temperature, SoC, ageing, etc.

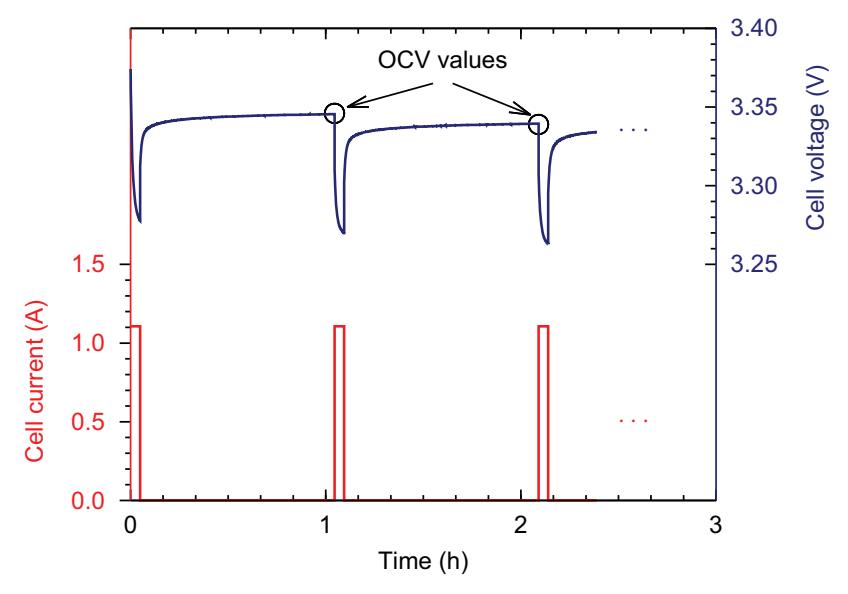

Figure 2. Measured voltage of an NMC cell showing the large relaxation times occurring after current interruption during a pulsed current test.

\section{A. OCV modeling}

A stated above, the OCV-SoC relationship is fundamental to describe the static behavior of the lithium-ion cell. Very often this relationship is inverted to estimate the cell $\mathrm{SoC}$ by the measurement of the OCV. The technique may be successful if the cell is in a really static condition (i.e. all the transients are expired) and the curve is not too flat, so that it can be inverted with small errors. An accurate measurement and modeling of the OCV-SoC curve is thus important for the construction of the overall battery model.

1) OCV measurement: The experimental measurement of the OCV is unfortunately a not trivial task, as the cell must reach the steady state before the voltage measurement value is collected. Fig. 2 shows the voltage response as a function of time of a lithium-ion cell with Nickel-Manganese-Cobalt (NMC) cathode. The time-domain response when the load is disconnected and the current drops to zero shows a very long transient that lasts for minutes and even hours. As the battery must be steady for the accurate measurement of the OCV, modeling a battery cell with an accurate OCV-SoC characteristic is a very long task, because each point of the characteristic calculated at a given $\mathrm{SoC}$ requires a long time to extract the corresponding OCV value. Usually, the suggested rest time is between 1 and $3 \mathrm{~h}$ for each point [16]. This measurement procedure is called PCT, just because the load current is pulsed at a fixed value for a given amount of time [17]. Each current pulse extracts a fixed amount of charge from the battery and thus a fixed SoC variation is determined by each pulse. The voltage value at the end of the rest time is considered to be the OCV value corresponding to that particular SoC value. The long experimental procedure, a few pulses of which are shown in Fig. 2, finally ends up with a curve like that reported in Fig. 3, which shows the OCV-SoC relationship of an NMC cell at three different temperatures. The curve is non linear, monotonic and can be inverted with acceptable errors. 


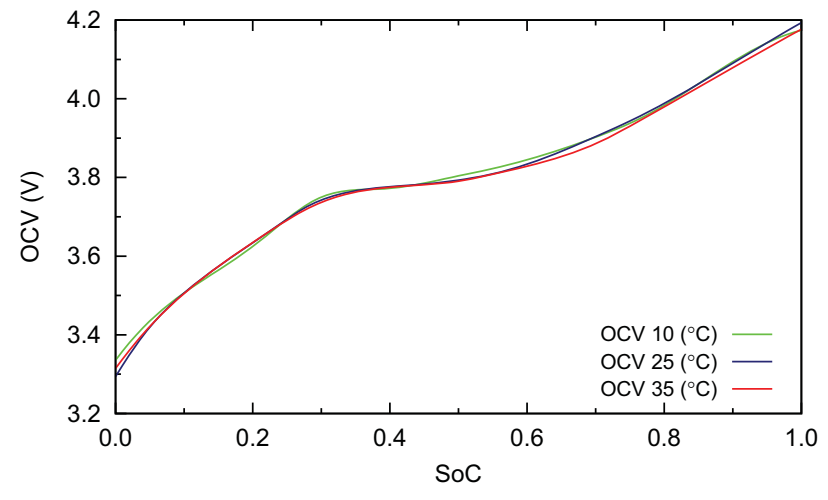

Figure 3. Open circuit voltage of an NMC measured at three different temperatures.

2) OCV model implementation: The availability of an accurate model of the battery allows a better control of it, as the battery response (e.g. the battery SoC) can be predicted with higher reliability. The BMSs accompanying a lithiumion battery, particularly the most advanced ones, usually implement some sort of modeling of the battery, trading off the model simplicity to the model accuracy. A very accurate and complex model of the cell requires computational resources that may be not available in some applications where the online real-time computation of the model is required. A very popular way of implementation of the OCV-SoC curve is by means of Look-up-Tables (LUTs), in which the function values are stored and read when the model is simulated. The other equivalent circuit parameters are also stored in LUTs, that may also include the temperature dependency of the parameters. The availability of rather large memory resources at low cost makes this approach cost effective. Another way to model the OCV-SoC curve is to approximate it with a mathematical function or superposition of functions. In that case, being the mathematical functions known, the SoC values are calculated on the fly over the hardware platform. A review of some of the possible functions used to model the OCV curves can be found in [13].

\section{B. Hysteresis in $O C V$}

The problem of the lithium battery modeling is further complicated by the experimental observation of hysteretic phenomena in some particular variants of the lithium chemistry, such as lithium-iron-phospate (LFP) batteries, where the OCVSoC characteristic shows a very pronounced hysteresis [17][19]. Here, the OCV-SoC curves are different when measured during the discharge or the charge phases. Fig. 4 shows the OCV measured on a $20 \mathrm{Ah} \mathrm{LFP} \mathrm{cell,} \mathrm{when} \mathrm{the} \mathrm{cell} \mathrm{is} \mathrm{first}$ fully discharged (bottom curve) and then fully recharged (top curve), spanning the SoC full range and describing the socalled major hysteresis loop. If the $\mathrm{SoC}$ is spanned in narrower ranges, the OCV describes minor loops always enclosed in the major one. Therefore, the flatness of the characteristic and the presence of hysteresis, combined together, make

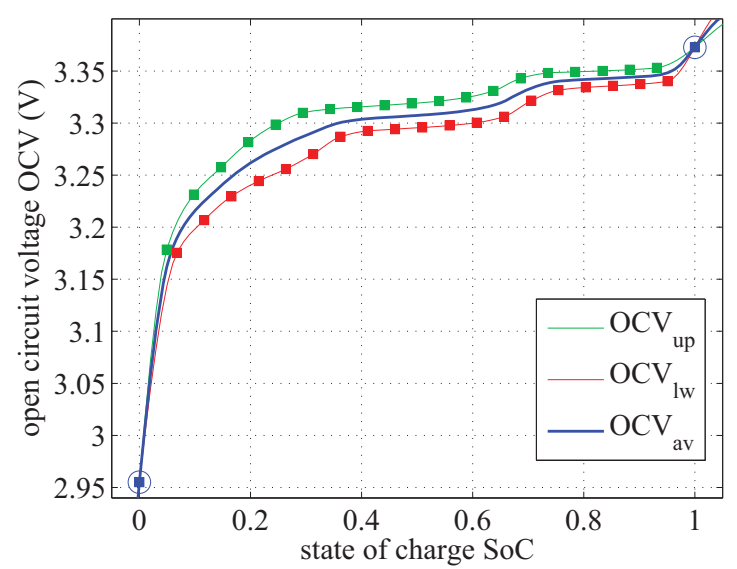

Figure 4. Open circuit voltage of an LFP cell showing a pronounced hysteresis between the discharge and charge curves [20].

practically impossible the extraction of the SoC from an OCV measurement.

In conclusion, a lithium ion cell model is necessary for a better exploitation of the battery, calling for a useful, reliable and accurate OCV model. This model requires to address issues of complex measurement procedures and hysteresis compensation.

\section{OCV HYSTERESIS CHARACTERIZATION AND MODELING}

A comprehensive OCV characterization of batteries showing hysteresis requires the measurement of several branches within the major loop in order to reconstruct any OCV evolution corresponding to the full history of the SoC. This result in a very time consuming procedure for a complete characterization of the OCV hysteresis behavior by means of the PCTs. A possible way to make the PCTs less cumbersome is to explore the dependence of the OCV value on the relaxation times, to possibly shorten the overall duration of the characterization procedure [21]. Ref. [22] investigates the variation of the OCV-SoC characteristics in an LFP cell as a function of the relaxation time. Fig. 5 shows the major hysteresis loop of an LFP loaded with a $1 \mathrm{C}$ current, calculated by taking the OCV points during relaxation at different times ranging from $1 \mathrm{~min}$ to $3 \mathrm{~h}$. It is evident how the gap between the discharging and the charging curves becomes narrower with time due to relaxation and tends to a limit value that defines the final hysteresis loop. It is important to note that $10 \mathrm{~min}$ is a time sufficient to extract a rather precise value of the final OCV, so that the PCT procedure may significantly be shortened. Thus, after having found a shorter way to extract the hysteretic OCV characteristic, we have to find a model for the hysteresis. Let us remind that the hysteresis major loop is the bound in which all the minor loops are confined.

To take into account hysteresis, the voltage source OCV shown in Fig. 1 can be considered as the sum of an average OCV (see the middle curve $\mathrm{OCV}_{a v}$ in Fig. 4 that represents 


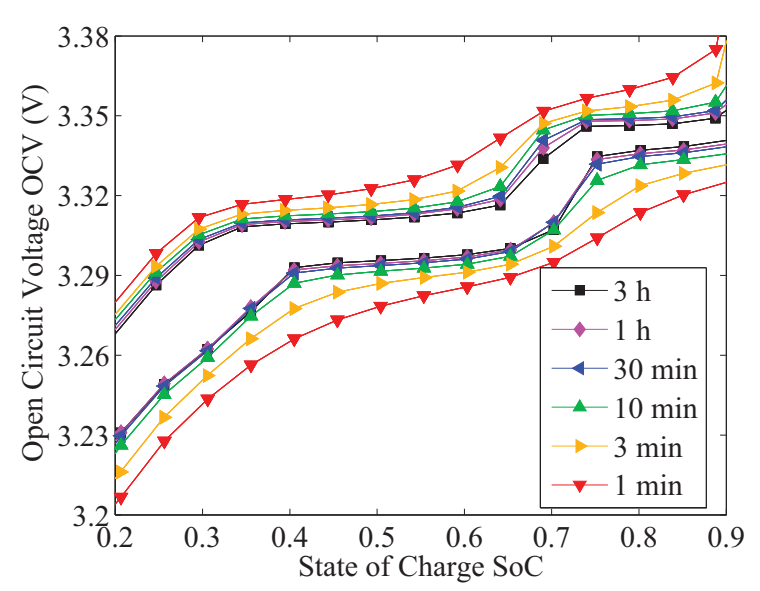

Figure 5. Major hysteresis loop in an LFP cell measured at different times ranging from $1 \mathrm{~min}$ to $3 \mathrm{~h}$ during relaxation. $10 \mathrm{~min}$ is a time sufficient to figure out the OCV final value [22].

the average OCV value) and a hysteresis generator $v_{H}$, the value of which accounts for all the hysteresis effects.

\section{A. One State Hysteresis (OSH) model}

One simple way to account for hysteresis is to model the phenomenon with one state system and consider the generic OCV evolution affected by a sort of relaxation in SoC, so that the OCV tends to reach the upper or lower bounds (the major loop curves in charge or discharge) after a "SoC constant" $\gamma$ analogous to the time constant found in the one state time relaxation phenomena [23]. Therefore, the hysteresis generator $v_{H}$ is easily calculated with a first order relaxation equation, provided that $\gamma$ is known. The model is very simple but needs an experimental procedure to identify $\gamma$, which may be very sensitive to the identification experiment. Ref. [17] has shown the application of the OSH model to $20 \mathrm{Ah} \mathrm{LFP}$ batteries, finding a very good fitting with rather small errors. The weak aspect of the approach is the dependence of $\gamma$ on the experiment with which it is identified.

\section{B. Preisach model with Everett function}

As hysteresis is a phenomenon deeply studied in magnetism, another model that has been applied to hysteresis in lithium batteries is the Preisach model, very popular for magnetic material modeling. The basic idea stands in considering hysteresis as composed by the superposition of elemental relays, the thresholds of which are distributed according to a specific distribution. Without going into much detail, it has been shown in [20] that the Preisach model can be identified with an experimental procedure that consists in the realization of FirstOrder-Reversal (FOR) branches, i.e. the experimental exploration of the OCV-SoC characteristics with discharge-charge loops that progressively decrease their amplitude from the full $100 \%$ SoC scale down to 0 . In this case the identification experiment is well defined, but the problem is finding the right Preisach distribution to be used to fit the hysteretic behavior.
The key is completing the identification procedure by using the Everett function $\mathcal{E}(\alpha, \beta)$, defined as

$$
\mathcal{E}(\alpha, \beta)=\frac{O C V_{\alpha}-O C V_{\alpha \beta}}{2}
$$

where $O C V_{\alpha}$ and $O C V_{\alpha \beta}$ are the $\mathrm{OCV}$ values measured along the FOR branches identified by the parameters $\alpha$ and $\beta$, the two SoC values that define the extrema of each FOR branch, as detailed in [24]. In this case, it has been shown that a generic OCV point can be calculated as a linear combination of the Everett function values in appropriate point of inversion. The identified Everett function can be stored in a LUT. Hysteresis is thus modeled with a low complexity algorithm, provided that the Everett function of the lithium cell under investigation is properly identified.

\section{Model comparison}

The above described models seems to be capable of properly modeling the hysteresis in OCV of an LFP cell. Let us report and discuss some experimental results with which the models are validated and compared to each other [24]. an LFP cell of $20 \mathrm{Ah}$ capacity has been subjected to several PCTs in a thermal chamber with a constant temperature of $298 \mathrm{~K}$. The OCV points are obtained with a sequence of current steps that determine a $5 \%$ SoC variation each. OSH model is identified by extracting the parameter $\gamma$ as a fitting parameter that minimizes the rms error between the experimental and the simulated data. The best found value is $\gamma=17.45$, but the value is found to be very sensitive to the fitted data, as expected. Instead, Preisach model is identified from the FOR branches described above, that span from $100 \%$ SoC to $0 \%$, increasing each time the branch depth with a $10 \%$ step. The Everett function values obtained in that coarse grid are then interpolated on a finer grid to obtain a better resolution. The OSH and Preisach models are then applied to fit the results that comes from three experiments. The first experiment is the major hysteresis loop and the OCV measured according to the above described procedure are compared to the OCV values calculated as output of the simulated models. The comparison is reported in Fig. 6.

The second experiment exerts the battery in a rather large minor loop traveling to the following sequence of SoC points: 100-25-75-25-100. The comparison is shown in Fig. 7.

Finally, a third experiment where the SoC points travel to the 100-40-60-40-100 sequence is carried out. The comparison between the model outputs and the experimental data is reported in Fig. 8.

All the experiments show the outstanding result that both the models account for hysteresis in the OCV of an LFP battery with a very good approximation. The errors are very limited in all the cases considered, as the rms error is always below $1.5 \%$. The minor loop experiments show that the models fit almost perfectly during the discharge branch of the OCV-SoC characteristic, whereas are less accurate during the recharge phases. However, the minor loop evolutions are reproduced very well by both the models. As far as a comparison between 


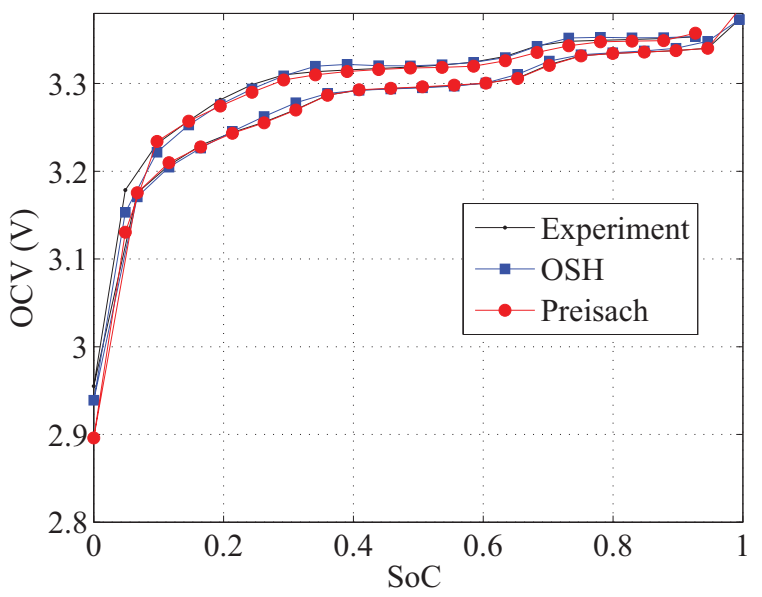

Figure 6. Comparison for the major loop [24].

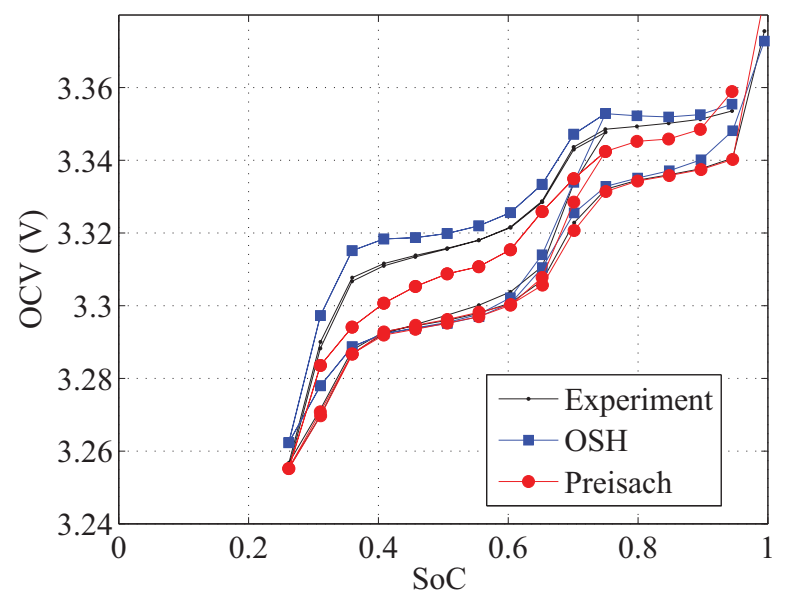

Figure 7. Comparison for one minor loop passing through the 100-25-75-25100 SoC points [24].

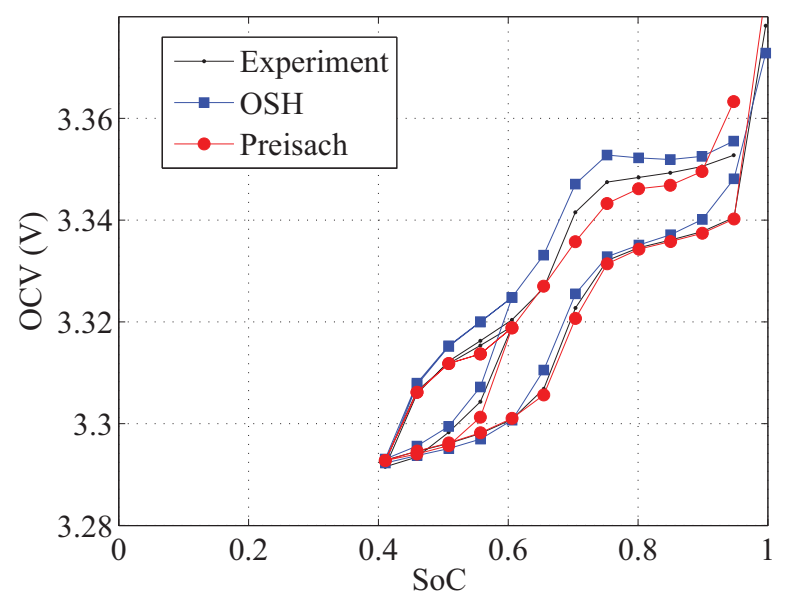

Figure 8. Comparison for one minor loop passing through the 100-40-60-40100 SoC points [24]. the models is concerned, no one of them outperforms the other, as the error performance is rather similar. However, it is important to note that the OSH outstanding results are obtained after an optimization process that allowed us to find the best fitting value of the parameter $\gamma$. Instead, the Preisach model has been identified with a procedure well defined and independent of any fitting procedure. This procedure allows the construction of the Everett function for the battery cell that is employed as is in any possible modeling experiment. Therefore, the latter model seems to us by far the most appropriate for the implementation of circuit models of lithium battery that may take into account also the hysteresis effect. Being the computation complexity rather low, i.e. only linear combinations of LUT-stored Everett function values are required, the Preisach model also seems appropriate for implementation on embedded platforms for the real-time onthe-fly estimation of a lithium battery behavior, as it will be required in ESSs for DC microgrid applications.

\section{CONCLUSIONS}

DC microgrid are emerging as an efficient way for distributing energy with a local grain. The fundamental component of a DC microgrid that allows the match between the generation and utilization rates of the energy is the energy storage system. ESSs based on high performance lithium-ion batteries need control algorithm aware of the battery behavior, which can be reproduced by an appropriate battery model. The model needs to be simple, computable with low complexity hardware, and must reproduce at best the terminal battery voltage. A circuit model with an electromotive force generator and a resistorcapacitor network is the most popular approach. The OCV generator depends on the battery SoC, so that its response needs to be experimentally characterized. Moreover, LFP batteries show hysteresis in their behavior. We have shown that the static OCV-SoC curve of a lithium-ion battery can be measured with pulsed current tests with acceptable characterization time and that the hysteresis can be modeled by adding a specific term in the OCV generator. Two models for the hysteresis characterization have been investigated and it has been shown that the Preisach model for magnetic hysteresis can successfully be applied to LFP batteries, provided that the Everett function identification approach is adopted. In this case, the Everett function is constructed by means of a well defined and standardized experimental procedure on the battery. Experiments show that both the models investigated reproduce major and minor hysteresis loops with very small errors. The Preisach model seems to be the most suited for hysteresis modeling for its superior features of independence on the data on which the model identification is carried out.

\section{REFERENCES}

[1] A. T. Elsayed, A. A. Mohamed, and O. A. Mohammed, "DC microgrids and distribution systems: An overview," Electric Power Systems Research, vol. 119, no. 0, pp. 407 - 417, 2015.

[2] H. Qian, J. Zhang, J.-S. Lai, W. Yu, Q. Hao, Z. Jianhui, L. Jih-Sheng, and I. T. on Power Electronics, "A High-Efficiency Grid-Tie Battery Energy Storage System," IEEE Transactions on Power Electronics, vol. 26, no. 3, pp. 886-896, Mar. 2011. 
[3] B. P. Roberts and C. Sandberg, "The Role of Energy Storage in Development of Smart Grids," Proceedings of the IEEE, vol. 99, no. 6 , pp. 1139-1144, Jun. 2011.

[4] M. S. Whittingham, "History, Evolution, and Future Status of Energy Storage," Proceedings of the IEEE, vol. 100, no. Special Centennial Issue, pp. 1518-1534, May 2012.

[5] S. Vazquez, S. M. Lukic, E. Galvan, L. G. Franquelo, and J. M. Carrasco, "Energy Storage Systems for Transport and Grid Applications," IEEE Transactions on Industrial Electronics, vol. 57, no. 12 , pp. 3881-3895, Dec. 2010

[6] J. Wang, P. Liu, J. Hicks-Garner, E. Sherman, S. Soukiazian, M. Verbrugge, H. Tataria, J. Musser, and P. Finamore, "Cycle-life model for graphite-LiFePO4 cells," Journal of Power Sources, vol. 196, no. 8, pp. 3942-3948, Apr. 2011.

[7] H. Rahimi-Eichi, U. Ojha, F. Baronti, and M.-Y. Chow, "Battery Management System: An Overview of Its Application in the Smart Grid and Electric Vehicles," IEEE Industrial Electronics Magazine, vol. 7, no. 2, pp. 4-16, Jun. 2013.

[8] J. D. Dogger, B. Roossien, and F. D. J. Nieuwenhout, "Characterization of Li-Ion Batteries for Intelligent Management of Distributed Grid-Connected Storage," IEEE Transactions on Vehicular Technology, vol. 26, no. 1, pp. 256-263, 2011.

[9] C. Weng, J. Sun, and H. Peng, "A unified open-circuit-voltage model of lithium-ion batteries for state-of-charge estimation and state-of-health monitoring," Journal of Power Sources, vol. 258, pp. 228-237, Jul. 2014.

[10] X. Hu, R. Xiong, and B. Egardt, "Model-Based Dynamic Power Assessment of Lithium-Ion Batteries Considering Different Operating Conditions," IEEE Transactions on Industrial Informatics, vol. 10 , no. 3, pp. 1948-1959, Aug. 2014.

[11] M. Chen and G. Rincon-Mora, "Accurate Electrical Battery Model Capable of Predicting Runtime and I-V Performance," IEEE Transactions on Energy Conversion, vol. 21, no. 2, pp. 504-511, Jun. 2006.

[12] F. Baronti, G. Fantechi, E. Leonardi, R. Roncella, and R. Saletti, "Enhanced model for Lithium-Polymer cells including temperature effects," in IECON 2010 - 36th Annual Conference on IEEE Industrial Electronics Society. IEEE, Nov. 2010, pp. 2329-2333.

[13] B. Pattipati, B. Balasingam, G. Avvari, K. Pattipati, and Y. Bar-Shalom, "Open circuit voltage characterization of lithium-ion batteries," Journal of Power Sources, vol. 269, pp. 317-333, Dec. 2014.
[14] S. Abu-Sharkh and D. Doerffel, "Rapid test and non-linear model characterisation of solid-state lithium-ion batteries," Journal of Power Sources, vol. 130, no. 1-2, pp. 266-274, May 2004.

[15] P. Buschel, T. Gunther, and O. Kanoun, "Distribution of relaxation times for effect identification and modeling of impedance spectra," in 2014 IEEE International Instrumentation and Measurement Technology Conference (I2MTC). IEEE, May 2014, pp. 901-904.

[16] M. A. Roscher and D. U. Sauer, "Dynamic electric behavior and open-circuit-voltage modeling of LiFePO4-based lithium ion secondary batteries," Journal of Power Sources, vol. 196, no. 1, pp. 331-336, 2011.

[17] F. Baronti, W. Zamboni, N. Femia, R. Roncella, and R. Saletti, "Experimental analysis of open-circuit voltage hysteresis in lithiumiron-phosphate batteries," in IECON 2013 - 39th Annual Conference of the IEEE Industrial Electronics Society. IEEE, Nov. 2013, pp. 6728-6733.

[18] M. A. Roscher, O. Bohlen, and J. Vetter, "OCV Hysteresis in Li-Ion Batteries including Two-Phase Transition Materials," International Journal of Electrochemistry, vol. 2011, pp. 1-6, 2011.

[19] H. Rahimi Eichi and M.-Y. Chow, "Modeling and analysis of battery hysteresis effects," in 2012 IEEE Energy Conversion Congress and Exposition (ECCE). IEEE, Sep. 2012, pp. 4479-4486.

[20] F. Baronti, N. Femia, R. Saletti, C. Visone, and W. Zamboni, "Hysteresis Modeling in Li-Ion Batteries," IEEE Transactions on Magnetics, vol. 50, no. 11, pp. 1-4, Nov. 2014.

[21] M. Petzl and M. A. Danzer, "Advancements in OCV Measurement and Analysis for Lithium-Ion Batteries," IEEE Transactions on Energy Conversion, vol. 28, no. 3, pp. 675-681, Sep. 2013.

[22] F. Baronti, W. Zamboni, R. Roncella, R. Saletti, and G. Spagnuolo, "Open-Circuit Voltage Measurement of Lithium-Iron-Phosphate Batteries," in Proc. of the 2015 International Instrumentation and Measurement Technology Conference - I2MTC 2015, 2015.

[23] G. L. Plett, "Extended Kalman filtering for battery management systems of LiPB-based HEV battery packs: Part 2. Modeling and identification,' Journal of Power Sources, vol. 134, no. 2, pp. 262-276, 2004.

[24] F. Baronti, N. Femia, R. Saletti, and W. Zamboni, "Comparing OpenCircuit Voltage Hysteresis Models for Lithium-Iron-Phosphate Batteries," in IECON 2014 - 40th Annual Conference of the IEEE Industrial Electronics Society, 2014, pp. 5635-5640. 